\title{
Relation between Trust Attitudes Toward Automation, Hofstede's Cultural Dimensions, and Big Five Personality Traits
}

\author{
Shih-Yi Chien, Michael Lewis \\ School of Information Sciences \\ University of Pittsburgh \\ Pittsburgh, PA 15260 USA \\ shc56@pitt.edu,ml@sis.pitt.edu
}

\author{
Katia Sycara \\ Robotics Institute \\ Carnegie Mellon University \\ Pittsburgh, PA 15213 USA \\ katia@cs.cmu.edu
}

\author{
Jyi-Shane Liu \\ Dept. of Computer Science \\ National ChengChi University \\ Taipei, Taiwan \\ jsliu@cs.nccu.edu.tw
}

\author{
Asiye Kumru \\ Dept. of Psycholoy \\ Özyeğin University \\ Istanbul, Turkey \\ asiye.kumru@ozyegin.edu.tr
}

Abstract - Automation has been widely used in interactions with smartphones, computers, and other machinery in recent decades. Studies have shown that inappropriate reliance on automation can lead to unexpected and even catastrophic results. Trust is conceived as an intervening variable between user intention and actions involving reliance on automation. It is generally believed that trust is dynamic and an individual's culture or personality may influence automation use through changes in trust. To better understand how cultural and individual differences may affect a person's trust and resulting behaviors, the present study examined the effects of cultural characteristics and personality traits on reported trust in automation in U.S., Taiwanese and Turkish populations. The results showed individual differences significantly affected human trust in automation across the three cultures.

\section{INTRODUCTION}

As society grows more dependent on the assistance of automation for an increasing number of tasks and endeavors, the problems of interacting with the technology become the limiting factor in the assistance such tools can provide. Human interaction with intelligent automation is an intricate process, requiring skilled operators and delicate system designs in order to effectively enhance overall performance. However, inappropriate reliance on automation can lead to catastrophic outcomes, such as the loss of cooling at Three Mile Island.

Prior research (Lee \& See, 2004) suggested that trust is an attitude, leading to intentions and resulting in user behaviors involving the automation use. In general, trust is not static but evolves over time as a result of the human operator's experience with the system. Trust dynamics evolve in 3 phases that characterize trust over time: trust formation, where trustors choose to trust trustees and potentially increase their trust over time; trust dissolution, where trustors decide to lower their trust in trustees after a trust violation has occurred; and trust restoration, where trust stops decreasing after a trust violation and is restored - potentially not to the same level as before the trust violation (Lee \& Moray, 1992).

Trust has been studied in a variety of disciplines, including social psychology, human factors, and industrial organizational psychology (Chien, Lewis, Hergeth, SemnaniAzad, \& Sycara, 2015; Lee \& Moray, 1992; Lee \& See, 2004; Mcknight, 2009; Sanders, Oleson, Billings, Chen, \& Hancock, 2011). Trust fluctuates between autonomous assistance and manual operation involving a variety of factors. It is generally believed that trust is dynamic and influenced by both endogenous (personality or culture) and exogenous (system reliability or task complexity) variables. For example, selfconfidence in an operator's ability to perform a task has been widely studied as a factor influencing trust, in which high self- confidence leads to lessened trust and decreased automation use (Lee \& Moray, 1992). However, later work (Moray, Inagaki, \& Itoh, 2000), where participants interacted with a higher level of automation, found that trust was influenced by properties of the system alone while self-confidence was influenced by operator personality traits.

\section{CULTURAL AND INDIVIDUAL DIFFERENCES}

Individual diversity in personality traits and cultural norms can have a significant impact on operator reliance on automation, since the perception of automation attributes, automation reliability and interpretation of results may differ considerably across operator populations. There has been little prior research on the relation of these characteristics on trust. Because we are interested in studying how individual differences affect the attitude of trust and hence intentions and behaviors, we face an important challenge in assuring that the measurement instruments are reliable across individual and cultural differences, so as to avoid confounding. Prior work, Big Five Personality Traits (John \& Srivastava, 1999), has provided a measure of personality based on five personality traits. In addition, Hofstede's Cultural Dimensions (Hofstede, 1991) and Cultural Syndromes (Triandis, 1996) provide complementary approaches to measuring cultural differences.

Validation studies have shown close agreement between Big Five Personality Traits scale and other measures of personality (John, Naumann, \& Soto, 2008) while Hofstede's Cultural Dimensions and Cultural Syndromes measures of culture have been shown to reliably discriminate between members of different cultures (Triandis \& Suh, 2002; Yoo, Donthu, \& Lenartowicz, 2011). 


\section{Big Five Personality traits}

Rotter (1967) suggested that people have different propensities to trust others (i.e., personality trait). Trait psychology identifies five major factors (Table 1) of individual differences with regard to personality, behaviors, and social attitudes (McCrae, 2000). The five-factor model of personality has been validated and supported by various works (John et al., 2008; McCrae, 2000), and it was adopted in this study to measure differences in personality traits.

TABLE 1. Five-factor model of personality

\begin{tabular}{|l|l|}
\hline \multicolumn{1}{|c|}{ Variable } & \multicolumn{1}{c|}{ Definition } \\
\hline Extraversion (E) & being talkative, energetic, or sociable \\
\hline Agreeableness (A) & being friendly, helpful, kind, or warm \\
\hline Conscientiousness (C) & being well organized or reliable \\
\hline Neuroticism (N) & being anxious, irritable, or anger \\
\hline Openness (O) & $\begin{array}{l}\text { being curious and seeking new } \\
\text { experience }\end{array}$ \\
\hline
\end{tabular}

\section{Cultural factors- Hofstede's cultural dimensions}

To examine the cultural effects on trust in automation, three of Hofstede's cultural dimensions were adopted in our study:

Power Distance $(P D)$ is defined as "the extent to which the less powerful accept and expect that power is distributed unequally (Hofstede, 1991)."

Individualism $(I D V)$ is "the degree of interdependence a society maintains among its members (Hofstede, 1991)."

Uncertainty Avoidance (UA) is defined as "the extent to which the members of a culture feel threatened by uncertain or unknown situations (Hofstede, 1991)."

\section{Cultural factors- Cultural Syndromes}

Cultural Syndromes (Triandis, 1996) provide an alternative way to characterize cultural differences (Figure 1) and overcome the major weakness of Hofstede's cultural mechanisms (Leung \& Cohen, 2011).

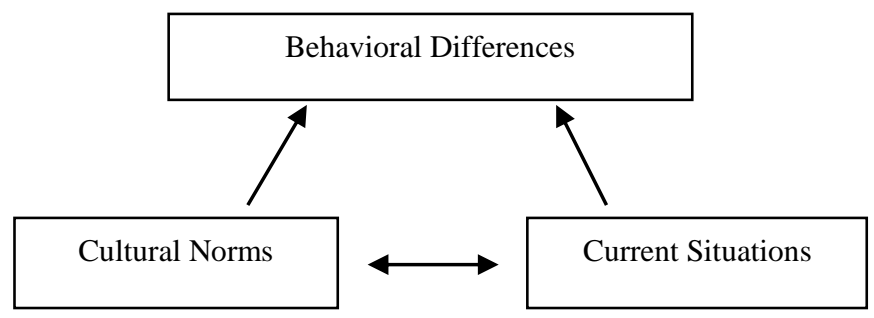

Figure 1. The relationships among cultural syndromes (norms), current task contexts, and resulting behaviors

Cultural Syndromes encompass the culture of Dignity, culture of Honor, and culture of Face:

Dignity Cultures are found in areas such as Western Europe and North America. People in Dignity cultures tend to make the "swift trust" assumption: others deserve to be trusted until they prove otherwise (Dirks, Lewicki, \& Zaheer, 2009).
Face Cultures are widespread in East Asian. In Face cultures, self-worth is stable and extrinsically derived based on social interactions with others, and what is important is the view that others have of you (Leung \& Cohen, 2011). Therefore, trust is high for those within the group and low for those outside.

Honor Cultures can be found in the Middle East Latin America, and Mediterranean countries along with Southern United States. Members of Honor culture tend to have low interpersonal and institutional trust.

These three mechanisms, Big Five Personality traits, Hofstede's cultural dimensions, and Cultural syndromes, were adopted to examine how personality traits and cultural dynamics may affect trust in automation.

\section{METHOD}

To examine cultural effects on trust in automation data sets were collected from the U.S., Taiwan and Turkey. These countries were selected because the contrasts they provided on Hofstede's dimensions (Figure 2) and Cultural Syndromes (categorizing US as a Dignity culture, Taiwan as a Face culture, and Turkey as an Honor culture).

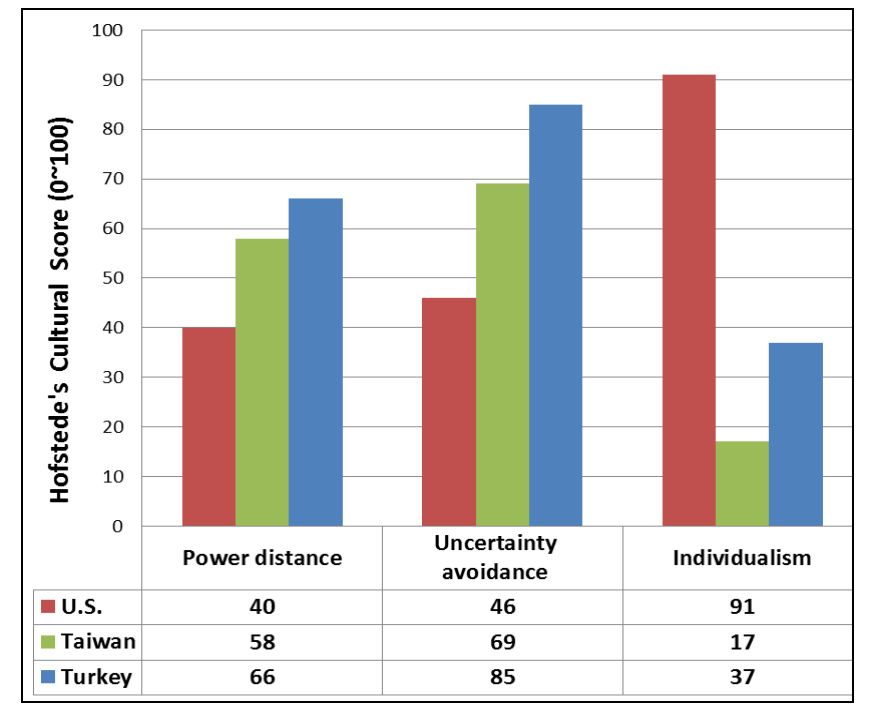

Figure 2. Country comparisons in Hofstede cultural dimensions

\section{Data collection}

The study was conducted in the U.S., Taiwan and Turkey, with 120 participants recruited in each country (i.e., a total of 360 responses were collected). The student participants were recruited from the University of Pittsburgh in the U.S. (avg. age=19.57), and National Chengchi University in Taiwan (avg. age $=21.60$ ), and Ozyegin University in Turkey (avg. age $=21.58)$. None had prior experience with air traffic control although most were frequent computer users.

The participants were asked to complete the Culture Trust Instrument (CTI; Chien et al., 2015; Chien, Semnani-azad, Lewis, \& Sycara, 2014). Data on Hofstede's (1991) cultural dimensions were collected using CVSCALE (Yoo et al., 2011) and on personality using the Big Five Inventory (John \& Srivastava, 1999) to provide a fuller picture of individual differences and their relation to the trust scale being developed. To avoid language issues, Chinese and Turkish versions of instruments were also used in our study. 


\section{Trust instrument}

The CTI consists of two scales: a general 32 item scale and a specific 18 item scale. The 32-item four-factor scale measures initial or general trust in automation without reference to any specific instance of automation. It can be thought of as a measure of predisposition to trust. The shorter 18-item, 3-factor specific scale references some particular instance of automation and is intended for use in experimental post tests and other cases where there is a desire to measure trust in some specific instances of automation.

Due to the page limit, results reported in this paper are for the general trust scale, which is composed of four dimensions:

Performance expectancy: an individual's beliefs that applying automation will enhance job performance.

Process transparency: the transparency of automation will affect an individual's degree of perceived difficulties in using it (i.e. how it functions).

Purpose influence: a person's knowledge of what the automation is supposed to do.

Task contexts: representing distinct elements of tasks, such as task complexity, risk, or workload.

\section{Cultural value scale $\&$ Big five inventory}

Cultural value scale (CVSCALE) was used to measure Hofstede's cultural values in the dimensions of power distance (PD), uncertainty avoidance (UA), and Individualism (IDV). The Chinese and Turkish versions of CVSCALE were adopted from (Gunkel, Schlaegel, \& Engle, 2014) and (Yoo et al., 2011) respectively.

Big five inventory (BFI), a 44-item five-factor model, was used to examine differences in personality traits. The Chinese and Turkish versions of BFI were adopted from (Leung, Wong, Chan, \& Lam, 2012) and (Vazsonyi, Ksinan, Mikuka, \& Jiskrova, 2015) respectively.

By directly scoring participants on CVSCALE and BFI, we were able to relate variations in trust and usage directly to dimensional profiles while classification by Cultural Syndromes can be inferred from nationality.

\section{RESULTS}

An ANOVA was used to examine the differences in general trust in automation, cultural characteristics, and personality traits among the U.S., Taiwanese, and Turkish populations. In addition, Pearson correlation coefficients were used to analyze association between general trust and cultural dimensions, and general trust and personality traits.

Due to the page limit, this study results focused only on the differences among cultural factors, personality traits, and trust ratings. The other measures (such as overall performance) remain to be describe elsewhere.

\section{General Trust using the CTI}

Significant differences were observed on general trust in automation across cultures $\left(\mathrm{F}_{2,357}=15.012, p<.001\right)$. Pairwise T-tests revealed differences between U.S. and Turkey $(p<.001)$, Taiwan and Turkey ( $p=.007)$, and U.S. and Taiwan $(\mathrm{p}=.048)$, in which the highest trust score was found in the U.S. (3.69) group and Turkish score was the lowest (3.33) with the Taiwanese rates falling in between (3.53), Figure 3 .

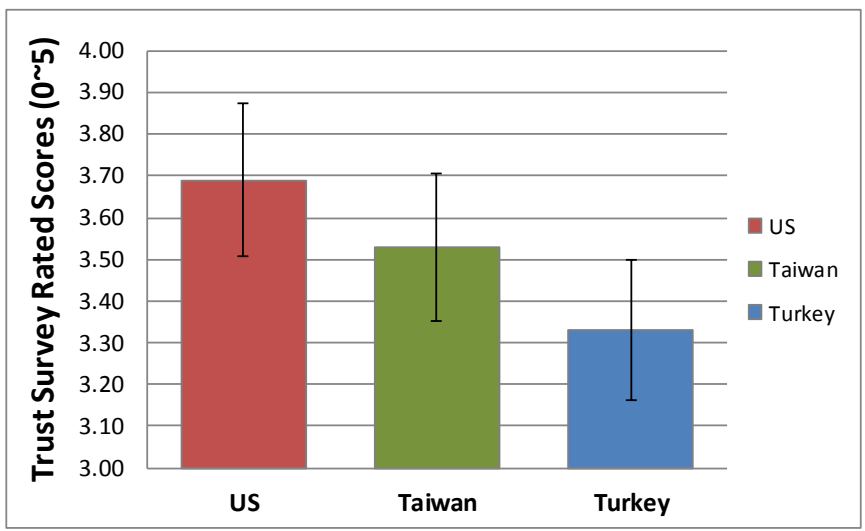

Figure 3. Trust scale rating comparisons: general trust in automation

\section{Cultural Value Scale (CVSCALE)}

The results of CVSCALE showed significant differences in all three cultural constructs, in which the U.S. group was higher in UA and IDV, and Taiwan population was higher in PD (Table 2). The results also revealed the differences between the Hofstede's original data, in which the data were collected from IBM employees between 1967 and 1973, and our collected data, in which the samples were drawn from student participants.

The results from Hofstede's original data suggested that the U.S. group had the lowest UA and Turkish population had the highest PD score among these three countries. However, our data showed the reverse results, in which the U.S. population now had the highest score in UA and Turkish participants had the lowest score in PD.

TABLE 2. CVSCALE rated scores across cultures

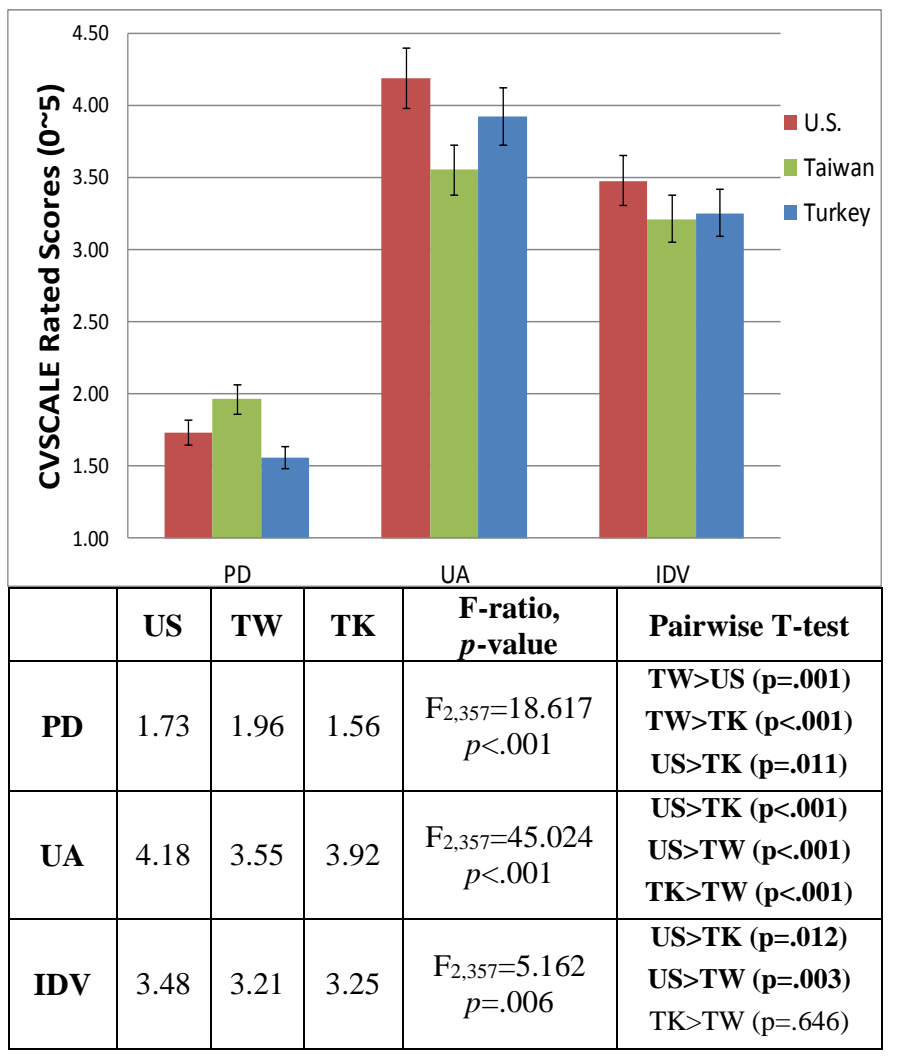




\section{Correlations for Trust Attitude and Cultural Dimensions}

Pearson correlation analysis (Table 3) for the cultural dimension and initial trust in automation showed highly significant differences between general trust and UA, and between general trust and IDV, which suggested an individual's initial trust was positively correlated to UA and IDV.

TABLE 3. Correlations for Trust Attitude and Cultural Dimensions

*. Correlation is significant at the 0.05 level (2-tailed).

**. Correlation is significant at the 0.01 level (2-tailed).

\begin{tabular}{|c|c|c|}
\hline \multirow{2}{*}{ PD } & \multicolumn{2}{|c|}{ General Trust } \\
\cline { 2 - 3 } & Pearson correlation & .077 \\
\cline { 2 - 3 } & Sig. Difference & .146 \\
\hline \multirow{2}{*}{ UA } & Pearson correlation & $.180^{* *}$ \\
\cline { 2 - 3 } & Sig. Difference &. $\mathbf{0 0 1}$ \\
\hline \multirow{2}{*}{ IDV } & Pearson correlation & $.194 * *$ \\
\cline { 2 - 3 } & Sig. Difference & $\mathbf{. 0 0 0}$ \\
\hline
\end{tabular}

Further analysis confirmed a positive correlation between UA and general trust, and between IDV and general trust in the U.S. population, whereas a positive correlation was also found between PD and initial trust, and between UA and initial trust in Turkish group; however, no significant difference was observed in Taiwan population (Table 4).

TABLE 4. Correlations for Trust Attitude and Cultural Dimensions in the U.S., Taiwan and Turkey populations

*. Correlation is significant at the 0.05 level (2-tailed).

**. Correlation is significant at the 0.01 level (2-tailed)

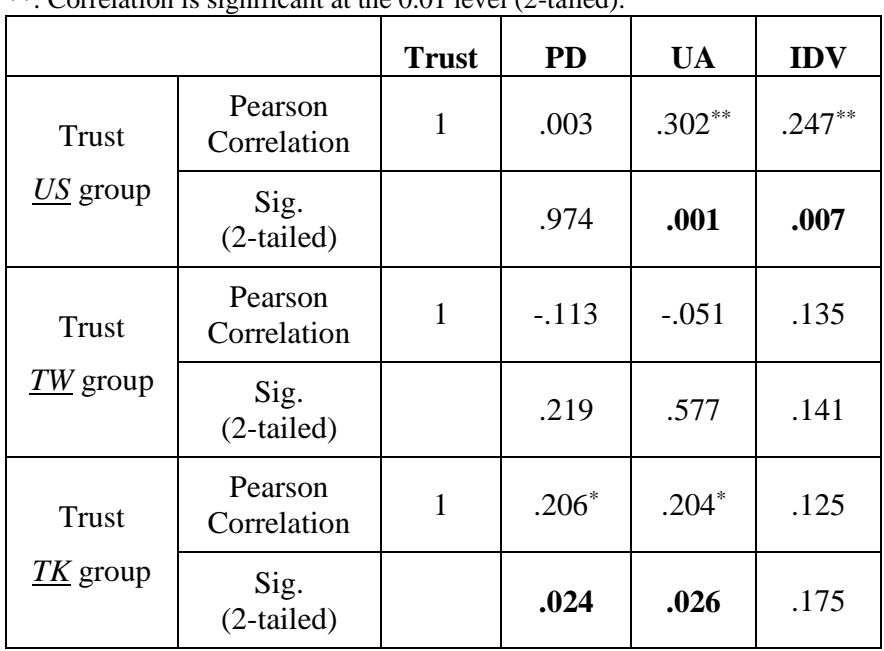

\section{Big Five Inventory (BFI)}

BFI was used to measure the differences in personality traits. An ANOVA showed significant differences between countries in all five measures (Table 5): Extraversion (E), Agreeableness (A), Conscientiousness $(\mathbf{C})$, Neuroticism (N), and Openness $(\mathbf{O})$.
TABLE 5. BFI scale rating comparisons

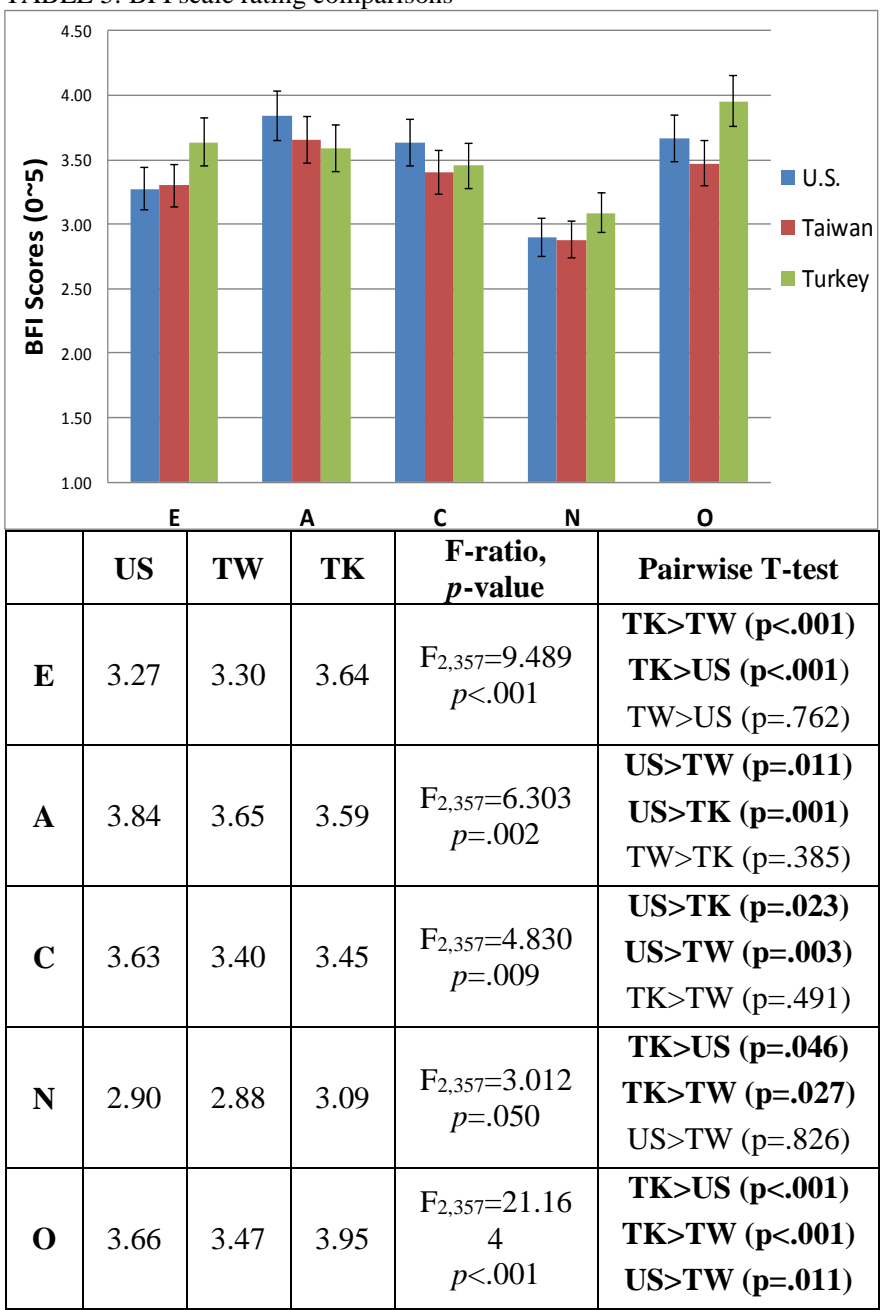

\section{Correlations for Trust and Personality Traits}

Pearson correlation analysis showed that only two dimensions, Agreeableness (A) and Conscientiousness (C), significantly correlated to an individual's initial trust. The results indicated that higher (A) or (C) values in an individual's personality traits resulted in higher initial trust in automation, Table 6.

TABLE 6. Correlations for Trust Attitude and Personality Traits **. Correlation is significant at the 0.01 level (2-tailed).

\begin{tabular}{|c|c|c|}
\hline \multirow{4}{*}{ E } & \multicolumn{2}{|c|}{ General Trust } \\
\cline { 2 - 3 } & Pearson correlation & .086 \\
\hline \multirow{3}{*}{$\mathbf{A}$} & Sig. Difference & .103 \\
\cline { 2 - 3 } & Pearson correlation & $.208^{* *}$ \\
\hline \multirow{3}{*}{$\mathbf{C}$} & Sig. Difference & .000 \\
\cline { 2 - 3 } & Pearson correlation & $.169 * *$ \\
\hline \multirow{3}{*}{$\mathbf{N}$} & Sig. Difference & .001 \\
\cline { 2 - 3 } & Pearson correlation & -.097 \\
\cline { 2 - 3 } & Sig. Difference & .065 \\
\hline \multirow{2}{*}{$\mathbf{O}$} & Pearson correlation & -.044 \\
\cline { 2 - 3 } & Sig. Difference & .407 \\
\hline
\end{tabular}




\section{DISCUSSIONS}

The present study examined the effects of cultural factors and personality traits on general trust in automation. Experimental studies were conducted in the U.S., Taiwan and Turkey to empirically measure how cultural characteristics and personality traits affected various aspects of initial trust in automation.

\section{General Trust}

According to cultural syndromes, due to the unstable social hierarchy, an individual from Honor culture (e.g., Turkey) in general has low initial trust to others, whereas the member of Dignity culture (e.g., U.S.) has high general trust and tend of trust others until proven otherwise. These cultural effects were confirmed, in which the U.S. population had the highest trust score and Turkish group scored the lowest, with Taiwanese population falling in between (Figure 3).

\section{Personality Traits and Cultural Factors}

Evaluations of the inter-relational aspects of personality and general trust showed that an individual with high trait of agreeableness or conscientiousness had increased trust in automation.

CVSCALE was used to measure the cultural values along Hofstede's cultural dimensions at the individual level. The mismatch between the Hofstede's original data and our collected data may be due to the differences of an individual's background, including age, education, knowledge, and prior experience with automation (Chien et al., 2014). In addition, since automation is increasingly being used in all aspects of our daily life (e.g., smartphone), this could significantly change people's beliefs about the use of automation.

The correlation analysis (Table 3) suggested that an individual will tend to rely on automated assistance when uncertainty is increased, especially in the U.S. and Turkish populations (Table 4, UA in the U.S. and Turkish groups). As predicted by Cultural Syndromes theory as IDV increased so did an initial willingness to trust in automation (Table 4, IDV in the U.S. population). Additionally, if using the automation was encouraged by the user's organization, the Turkish participants were more likely to trust the automation (Table 4, PD in Turkey population). However, there was no universal pattern across three cultures (Table 4).

The main objective of the present study was to examine the extent to which individual differences (culture and personality) were able to affect the formation of trust in automation. In future analyses, we will examine the combined effect of these three factors (trust, culture and personality) through empirical studies.

\section{ACKNOWLEDEMENTS}

This research has been sponsored by AFOSR FA9550-131-0129. The authors greatly acknowledge Ms. Ju-Chun Wei and Ms. Wan-Ching Hsieh at the University of Pittsburgh for translating the trust survey into Chinese, and Dr. Gizem Arikan at Özyeğin University for translating the trust instrument into Turkish.

\section{REFERENCES}

Chien, S., Lewis, M., Hergeth, S., Semnani-Azad, Z., \& Sycara, K. (2015). Cross-Country Validation of a Cultural Scale in Measuring Trust in Automation. Proceedings of the Human Factors and Ergonomics Society Annual Meeting.

Chien, S., Semnani-azad, Z., Lewis, M., \& Sycara, K. (2014). Towards the Development of an Inter-cultural Scale (pp. 3546). Springer International Publishing.

Dirks, K., Lewicki, R., \& Zaheer, A. (2009). Reparing Relationships Within and Between Organizations: Building A Conceptual Foundation. Academy of Management Review, 34(1), 68-84.

Gunkel, M., Schlaegel, C., \& Engle, R. L. (2014). Culture and a Cascading Model of Emotional Intelligence: An Exploratory Analysis. Multinational Enterprises, Markets and Institutional Diversity.

Hofstede. (1991). Cultures And Organizations - Software of the Mind. Development, 1-29. Retrieved from

John, O., Naumann, L., \& Soto, C. (2008). Paradigm shift to the integrative Big Five Trait taxonomy. Handbook of Personality: Theory and Research, 114-158.

John, O., \& Srivastava, S. (1999). The Big Five trait taxonomy: History, measurement, and theoretical perspectives. Handbook of Personality, (510).

Lee, J., \& Moray, N. (1992). Trust, control strategies and allocation of function in human-machine systems. Ergonomics, 35(10), $1243-1270$.

Lee, J., \& See, K. (2004). Trust in automation: designing for appropriate reliance. Human Factors, 46(1), 50-80.

Leung, A. K. Y., \& Cohen, D. (2011). Within-and between-culture variation: Individual differences and the cultural logics of honor, face, and dignity cultures. Journal of Personality and Social Psychology, 100(3), 507-526.

Leung, D. Y., Wong, E. M., Chan, S. S., \& Lam, T. (2012). Psychometric properties of the Big Five Inventory in a Chinese sample of smokers receiving cessation treatment: A validation study. Journal of Nursing Education and Practice, 3(6), 1-10.

McCrae, R. R. (2000). Trait Psychology and the Revival of Personality and Culture Studies. American Behavioral Scientist, 44(1), 10-31.

Mcknight, H. (2009). Trust in technology : development of a set of constructs and measures.

Moray, N., Inagaki, T., \& Itoh, M. (2000). Adaptive Automation, Trust, and Self-Confidence in Fault Management of TimeCritical Tasks. Experimental Psychology: Applieed, 6(1), 4458.

Rotter, J. B. (1967). A new scale for the measurement of interpersonal trust. Journal of Personality.

Sanders, T., Oleson, K. E., Billings, D. R., Chen, J. Y. C., \& Hancock, P. A. (2011). A Model of Human-Robot Trust Theoretical Model Development. Human Factors.

Triandis, H. (1996). The psychological measurement of cultural syndromes. American Psychologist.

Triandis, H., \& Suh, E. (2002). Cultural influences on personality. Annual Review of Psychology.

Vazsonyi, A. T., Ksinan, A., Miku??ka, J., \& Jiskrova, G. (2015). The Big Five and adolescent adjustment: An empirical test across six cultures. Personality and Individual Differences, 83, 234-244. http://doi.org/10.1016/j.paid.2015.03.049

Yoo, B., Donthu, N., \& Lenartowicz, T. (2011). Journal of International Consumer Marketing Measuring Hofstede 's Five Dimensions of Cultural Values at the Individual Level : Development and Validation of CVSCALE Measuring. Journal of International Consumer Marketing, 23(August 2011), 193-210. 